\title{
Effect of Anthropometric Features on the Severity of Lumbar Disk Herniation
}

\author{
Dong Yeob LEE and Sang-Ho LEE
}

Department of Neurosurgery, Wooridul Spine Hospital, Seoul, R.O.K.

\begin{abstract}
Normal standing body height, body weight, and body mass index were measured in 256 patients with severe lumbar disk herniation who underwent surgery and compared with those of matched controls with mild lumbar disk herniation who showed improvement of symptoms after conservative treatment. Statistical analysis was performed using the paired sample $t$ test and analysis of covariance test. Body weight and body mass index were significantly higher in women with severe lumbar disk herniation than in those with mild lumbar disk herniation $(p=0.01$ and $p=0.01$, respectively), but not in men. Standing body height showed no significant difference between patients with severe and mild lumbar disk herniations in both sex groups. Differences in body weight and body mass index may be key factors distinguishing the development of severe lumbar disk herniation from that of mild lumbar disk herniation in women.
\end{abstract}

Key words: lumbar vertebra, intervertebral disk, body mass index, obesity

\section{Introduction}

Anthropometric features, such as standing body height and/or body mass index (BMI), may be associated with the development of lumbar disk herniation, in terms of both anatomical expression and development of symptomatology requiring surgery..$^{1,3-5,7,8,12,13)}$ Most studies of the anthropometric analysis of lumbar disk herniation compared patients with lumbar disk herniation with healthy controls.

The present case control study investigated the associations between anthropometric features, such as standing body height, body weight, and BMI, and severity of lumbar disk herniation in patients who underwent decompressive surgery for severe lumbar disk herniation compared with patients who underwent successful conservative treatment for mild lumbar disk herniation.

\section{Materials and Methods}

This study included 256 patients aged 20 to 69 years who underwent decompressive surgery (conventional open discectomy or percutaneous endoscopic discectomy) for single-level lumbar disk herniation

Received March 25, 2008; Accepted October 28, 2008 from January 2005 to June 2005. The inclusion criteria were as follows: Single-level lumbar disk herniation with nerve root compression demonstrated by computed tomography (CT) and magnetic resonance (MR) imaging, radicular symptoms and/or back pain consistent with the radiologic findings, and unsuccessful conservative therapy for more than 6 weeks. Patients who underwent emergent surgery for severe neurological deficits, such as foot drop, cauda equina syndrome, or intractable leg pain, were also included in this study irrespective of the duration of conservative treatment. The exclusion criteria were as follows: Multi-level surgery, foraminal and/or extraforaminal disk herniation, previous history of spinal surgery, and medical disease, such as diabetes mellitus or thyroid disease.

The 256 control subjects were selected from 1537 patients who underwent successful conservative treatment for lumbar disk herniation in 2005. To exclude confounding factors, paired matching was performed by one author (D. Y. Lee), ${ }^{2,10}$ to provide same sex and similar age (differing by no more than \pm 10 years). The inclusion criteria were as follows: Single-level mild lumbar disk herniation without nerve root compression on CT and/or MR imaging, back pain and/or radicular symptoms consistent with the radiologic findings, and marked improvement of symptoms after conservative treatment (physical therapy and non-steroid anti-inflammatory 
drug medication for 6 weeks with or without epidural steroid injection). The exclusion criteria were as follows: Multi-level lumbar disk herniation, foraminal and/or extraforaminal disk herniation, previous history of spinal surgery, and medical disease such as diabetes mellitus or thyroid disease.

Normal standing body height and weight recorded in the clinical chart were evaluated. The mean values of standing body height $(\mathrm{cm})$, body weight $(\mathrm{kg})$, and BMI (weight/height ${ }^{2} ; \mathrm{kg} / \mathrm{m}^{2}$ ) were compared. Statistical analysis was performed using the paired sample $t$ test and analysis of covariance (ANCOVA) test with age as a covariate. $P$ values of less than 0.05 were considered significant.

\section{Results}

There were 150 men included in each of the study and control groups. No significant difference was found in mean age (paired sample t test, $p=0.78$ ), intervertebral disk level affected (chi-square test, $p$ $=0.95$ ), standing body height (paired sample $t$ test, $p$ $=0.20 ;$ ANCOVA test, $p=0.20$ ), body weight (paired sample t test, $p=0.81$; ANCOVA test, $p=$ 0.84 ), and BMI (paired sample $t$ test, $p=0.80$; ANCOVA test, $p=0.81$ ) between the groups (Table 1).

There were 106 women included in each of the study and control groups. No significant difference was found in age (paired sample t test, $p=0.62$ ), intervertebral disk level affected (chi-square test, $\mathrm{p}=$ 0.43), and standing body height (paired sample t test, $\mathrm{p}=0.95$; ANCOVA test, $\mathrm{p}=0.75$ ) between the groups. However, the study group had a significantly higher body weight than the control group (paired sample $t$ test, $p=0.01$; ANCOVA test, $p=0.01$ ), which resulted in a significantly higher BMI in the study group than in the control group (paired sample t test, $p=0.01$; ANCOVA test, $p=0.01$ ) (Table 2).

\section{Discussion}

Several European epidemiologic studies have suggested that anthropometric features, i.e., BMI and standing body height, have an association with the development of lumbar disk herniation. ${ }^{1,3-7,11-13)}$ Anthropometric measurements in patients with lumbar disk herniation found that men with a height of 180 $\mathrm{cm}$ or more have a relative risk of 2.3 and women with a height of $170 \mathrm{~cm}$ or more a relative risk of 3.7 , compared with those who are more than $10 \mathrm{~cm}$ shorter. ${ }^{5)}$ Also, increased BMI was an independent risk factor for herniated lumbar disk in men. Therefore, height and BMI might be important contributors to the herniation of the lumbar intervertebral disk. The relationship between tall stature and facet joint asymmetry may also be important in lumbar disk herniation. Patients with lumbar disk hernia-

Table 1 Results of matched analysis in men

\begin{tabular}{|c|c|c|c|c|c|c|c|c|}
\hline & \multirow{2}{*}{$\begin{array}{c}\text { Total } \\
\text { number }\end{array}$} & \multirow{2}{*}{ Age (years) } & \multicolumn{3}{|c|}{ Disk level } & \multirow{2}{*}{ Height $(\mathrm{cm})$} & \multirow{2}{*}{ Weight (kg) } & \multirow{2}{*}{ BMI $\left(\mathrm{kg} / \mathrm{m}^{2}\right)$} \\
\hline & & & L3-4 & L4-5 & L5-S1 & & & \\
\hline Study group* & 150 & $42.6 \pm 11.0(21-69)$ & 8 & 95 & 47 & $171.2 \pm 5.9(156-185)$ & $70.3 \pm 10.9(47-109)$ & $24.0 \pm 3.2(17.3-32.3)$ \\
\hline Control group ${ }^{\dagger}$ & 150 & $42.2 \pm 10.9(21-67)$ & 7 & 97 & 46 & $172.1 \pm 5.5(161-188)$ & $70.7 \pm 11.4(53-100)$ & $23.9 \pm 3.8(18.4-28)$ \\
\hline p Value & & $0.78^{\ddagger}$ & $0.95^{\S}$ & & & $0.20^{\ddagger}, 0.20^{||}$ & $0.81^{\ddagger}, 0.84 \|$ & $0.80^{\ddagger}, 0.81 \|$ \\
\hline
\end{tabular}

*Patients with severe lumbar disk herniation who underwent decompressive surgery. ${ }^{\dagger}$ Patients with mild lumbar disk herniation who showed improvement after conservative treatment. ${ }^{\ddagger}$ Paired sample t test, ${ }^{\S} \mathrm{chi}$-square test, $\|$ analysis of covariance test with age as a covariate. BMI: body mass index.

Table 2 Results of matched analysis in women

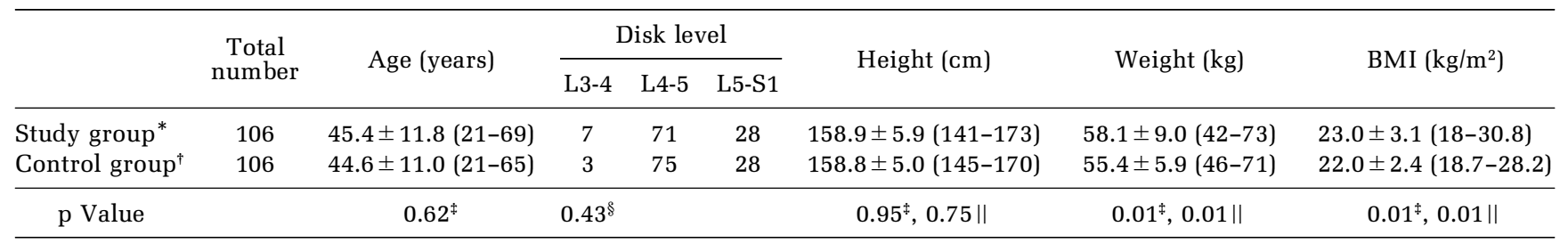

${ }^{*}$ Patients with severe lumbar disk herniation who underwent decompressive surgery. ${ }^{\dagger}$ Patients with mild lumbar disk herniation who showed improvement after conservative treatment. ${ }^{\ddagger}$ Paired sample t test, ${ }^{\S} \mathrm{chi}$-square test, $\|$ analysis of covariance test with age as a covariate. BMI: body mass index. 
tion show asymmetry and sagittalization of the facet joints, and these changes were more evident in taller patients. ${ }^{6)}$ Increased BMI and tall stature appear to be more strongly associated with the development of severe lumbar disk herniation requiring surgery, ${ }^{1)}$ and patients operated on for lumbar disk herniation are typically tall, with a relatively high BMI, and are aged between 19 and 48 years. ${ }^{3)}$ Therefore, higher body weight and height are generally supposed to increase vertebral column loading, resulting in overstraining of the intervertebral disk. ${ }^{11)}$ The relationship between increased body weight and/or tall height and occurrence of lumbar disk herniation has been rarely reported from Asian countries. In our previous study analyzing Korean who underwent surgery for lumbar disk herniation, men with disk herniation were significantly taller than ordinary population $(171.6 \pm 6.4$ vs. $170.0 \pm 5.6, \mathrm{p}<0.001)$ and women with disk herniation was significantly heavier than ordinary population $(58.9 \pm 9.6$ vs. $57.2 \pm 7.8, \mathrm{p}=0.029) .{ }^{12)}$ Most of these anthropometric analyses of lumbar disk herniation compared patients with lumbar disk herniation with the general population..$^{1,3-8,10,12,13)}$

In the present study, patients with mild lumbar disk herniation who showed marked improvement of symptoms after conservative treatment were used as the control group. The present study found that anthropometric features were not a key factor distinguishing development of severe lumbar disk herniation requiring surgery from mild lumbar disk herniation in men. In contrast, a significantly higher body weight and BMI were associated with severe lumbar disk herniation in women. Therefore, body weight and BMI might be considered key factors distinguishing the development of severe lumbar disk herniation from that of mild lumbar disk herniation in women. In Korea, men are more frequently involved in severe daily physical activities, such as heavy lifting or sports, than women, and these activities are considered important risk factors for the development of severe lumbar disk herniation requiring surgery. Therefore, greater body weight and higher BMI may affect the development of severe lumbar disk herniation more prominently in women than in men. The present study excluded patients with diabetes mellitus or thyroid disease because these diseases may affect body weight and BMI.9) We performed paired matching for age (differing by no more than \pm 10 years), ${ }^{2,10}$ ) so the mean ages of the two groups were not significantly different. Nevertheless, to exclude the possibility that age still affected the result of the current study, we also performed the ANCOVA test with age as a covariate.

The present study analyzed patients with lumbar disk herniation who underwent surgical treatment or conservative treatment. Therefore, anthropometric analysis of Koreans with healthy backs might further reveal the significance of anthropometric determinants on the occurrence of lumbar disk herniation. We cannot extrapolate the conclusion of this study concerning the influence of anthropometric features on the severity of lumbar disk herniation to other races including Caucasians because of different body habitus and life style.

\section{Acknowledgment}

This study was supported by a grant from the Wooridul Spine Foundation.

\section{References}

1) Böstman OM: Body mass index and height in patients requiring surgery for lumbar intervertebral disc herniation. Spine 18: 851-854, 1993

2) Dora C, Schmid MR, Elfering A, Zanetti M, Hodler J, Boos N: Lumbar disk herniation: do MR imaging findings predict recurrence after surgical diskectomy? Radiology 235: 562-567, 2005

3) Dudek H, Michno T, Michalski J: [Anamnestic and anthropometric assessment of patients with herniated lumbar intervertebral disc]. Chir Narzadow Ruchu Ortop Pol 64: 619-625, 1999 (Pol, with Eng abstract)

4) Gyntelberg F: One-year incidence of low back pain among male residents of Copenhagen aged 40-59. Dan Med Bull 21: 30-36, 1974

5) Heliövaara M: Body height, obesity, and risk of herniated lumbar intervertebral disc. Spine 12: 469-472, 1987

6) Karacan I, Aydin T, Sahin Z, Cidem M, Koyuncu H, Aktas I, Uludag M: Facet angles in lumbar disc herniation: their relation to anthropometric features. Spine 29: 1132-1136, 2004

7) Kelsey JL: An epidemiological study of acute herniated lumbar intervertebral discs. Rheum Rehabil 14: 144-159, 1975

8) Lee DY, Ahn Y, Lee SH: Anthropometric analysis of patients with lumbar disc herniation. Korean Journal of Spine 1: 477-480, 2004

9) Lee JH, Song CH, Yum KS, Kim KS, Nam SW, Han JY, Jeong GW, Sun HS: Age associated changes in body mass index and body fat distribution. Journal of Korean Academy of Family Medicine 24: 1010-1016, 2003

10) Park JB, Chang H, Kim KW, Park SJ: Facet tropism: a comparison between far lateral and posterolateral lumbar disc herniations. Spine 26: 677-679, 2001

11) Pietilä TA, Stendel R, Kombos T, Ramsbacher J, Schulte $\mathrm{T}$, Brock $\mathrm{M}$ : Lumbar disc herniation in patients up to 25 years of age. Neurol Med Chir (Tokyo) 41: 340-344, 2001

12) Pope MH, Bovins T, Wilder WG, Frymoyer JW: The 
relationship between anthropometric, postural, muscular, and mobility characteristics of male aged 18-55. Spine 10: 644-648, 1985

13) Rissanen A, Heliövaara $M$, Knekt $P$, Reunanen A, Aromaa A, Maatela J: Risk of disability and mortality due to overweight in a Finnish population. Br Med J 13: 835-837, 1990

Address reprint requests to: Sang-Ho Lee, M.D., Department of Neurosurgery, Wooridul Spine Hospital, 47-4 Chungdam-dong Gangnam-gu, Seoul 135-100, R.O.K.

e-mail: shlee@wooridul.co.kr

\section{Commentary}

This is an excellent study from a superb spine group in one of the most prominent spine hospitals in Seoul. Dr. Lee and his group designed and here report a clever, novel, and innovative hypothesis. They have reviewed the effect of anthropometric features on the severity of lumbar disk herniation in a case controlled study for a large number of patients seen in their institution over just a six month period. They compared height, weight, and BMI in single-level lumbar disk herniation patients of both sexes, using as a control group individuals who improved with conservative therapy. Exclusion criteria involved patients with multilevel disease, foraminal or extraforaminal disk herniation. In the male cohort, BMI, height, and weight were not associated with worsening lumbar disk herniation requiring surgery. However, in women, BMI and weight, but not height, were associated with severe lumbar disk herniation.

Consideration of the method reveals several areas of interest. The authors selected control subjects by paired age and sex matching. This was followed by inclusion into the control group if the patient had mild disk herniation without nerve root compression on CT or MRI. Control matching can often lead to selection bias, and we note that the inclusion criteria are not entirely similar to that in the surgical group. There is some ambiguity as to whether the disk disease was diagnosed on CT or MRI. This is an important point if the goal is to compare mild versus severe disk herniation, as imaging interpretation may differ depending on the study type.

Physical therapy is often recommended for six weeks with NSAIDS with or without epidural steroid injections. A standardized physical therapy regimen may lend strength to the study as well. Also of note, information on outcomes regarding patients who underwent surgery, and the method of diskectomy, would be interesting. This study attempts to parse out which patients with mild disk herniation should be more closely monitored in the daily clinical setting. It may be beneficial to also compare waist circumference in a similar study, as we are currently finding in the U.S. population that this seems to have a significant association with diseases associated with elevated BMI. Dr. Lee's group readily recognizes that this cohort may not be comparable to other races due to differences in body habitus and lifestyle, however, further evaluation in a prospective randomized manner could provide beneficial information as to which patients with mild disk herniation progress to severe disk herniation and would require surgical intervention.

All these minor considerations aside, this is an excellent early study of a clever idea and we applaud the authors for their scientific rigor and their elegant presentation of the data. We look forward to learning more from then, considering their vast experience and large patient population.

Pulak RAY, M.D. and Christopher M. LOFTUS, M.D., D.H.C. (Hon.), F.A.C.S. Department of Neurological Surgery Temple University School of Medicine Philadelphia, Pennsylvania, U.S.A.

Dr. Lee retrospectively examined the effect of anthropometric features in patients with lumbar disk herniation using a good study design. Among the many anthropometric studies on the prevalence of lumbar disk herniation comparing patients and healthy subjects, the unique point of this study is that patients with mild lumbar disk herniation who had marked relief of symptoms following conservative treatment were used as the controls.

This study showed statistically significant difference in body weight and BMI between the patients with surgical intervention and those with conservative treatment in women; higher body weight and BMI were well correlated to the severity of lumbar disk herniation. On the other hand, no significant difference was indicated in men in this study.

Sex-associated habitual and social differences in Korea cannot completely persuade readers worldwide and racial differences will not be evaluated precisely, however, we can still assume that body weight and BMI are reliable predicting factors in severity or in surgical indication of lumbar disk herniation. Furthermore, an investigation on the recurrence of lumbar disk herniation is expected from the anthropometric point of view for patient's education.

Satoshi TANI, M.D. Department of Neurosurgery The Jikei University School of Medicine Tokyo, Japan 